\title{
FPGA-based Hierarchical Finite-States Predictive Control for PMSM drives
}

\author{
Matteo Carraro*, Mauro Zigliotto*, Luca Peretti ${ }^{\dagger}$ \\ *Department of Engineering and Management, University of Padova, Vicenza, Italy, ${ }^{*}$ ABB Corporate Research, Dept. of \\ Power Technologies, Forskargränd 7,Västerås, Sweden, email: luca.peretti@se.abb.com
}

Keywords: Predictive Control, PMSM drives, FPGA

\begin{abstract}
The aim of the research is the development of a finite-state predictive speed and current control algorithm for a permanent magnet synchronous motor (PMSM). The high computational requirement is one of the main limitations in the calculation and application of the switching states to the inverter, but an alternative control strategy is here proposed. Preliminary simulation and experimental and results obtained with a FPGA-based control board prove the feasibility of the predictive control architecture, both in transient and steadystate conditions.
\end{abstract}

\section{Introduction}

In view of the increasing demand of energy-efficient drives, predictive control techniques are arousing keen interest in the research area of advanced control techniques for power electronic converters and electric drives. The Model Predictive Control (MPC) may be a promising alternative to either the conventional field-oriented control (FOC) or the direct torque control (DTC) [1], [2], [3]. The main difference between predictive control and traditional strategies is the pre-calculation of the system behaviour [4], [5]. Many nonlinear constraints can be included in the control law, but a high number of calculations may be required to compute and test all the possible configurations [6]. This could penalise the on-line solving of the predictive control law, limiting the implementation only to a finite set of candidate voltages tested on the prediction model of the electrical machine (the so-called direct predictive control, DPC). However, the latest developments in the computational power of the DSPs and the introduction of FPGA platforms allow the implementation of more complex real-time predictive control techniques.

This paper deals with the design of a DPC version which integrates the speed and current control for a PMSM drive [7]. The attempt is to enhance the original DPC by the inclusion of a hierarchical decisional structure in the control law. The proposed control structure is named Hierarchical Direct Predictive Control (HDPC). The minimisation of a quadratic cost function is replaced by a multi-level decisional structure, where the next control input within a finite set of voltage vector is selected by successive refinements. While maintaining a fast dynamic, this solution allows a straightforward implementation of the state variable limits. A first-order disturbance observer is also included in the drive model to estimate the load torque, in order to avoid unpredicted steady-state speed errors due to load torque variations.

\section{Theory of operations}

A PMSM fed by a three-phase voltage source inverter is considered for the system modelling. Each of the three-phase legs of the inverter is characterized by two switches that connect the voltage source $U_{D C}$ of the DC link with the neutral point $N$, therefore the voltage in each phase is $\pm U_{D C} / 2 \mathrm{~V}$. The voltage configurations generated without modulation are limited to $N_{S}=2^{3}$ combinations, and each state of the inverter is represented in space vector form as:

$\boldsymbol{u}_{s i}=\frac{2}{3}\left(u_{u N}+u_{v N} e^{j \frac{2 \pi}{3}}+u_{w N} e^{j \frac{4 \pi}{3}}\right) \quad i=0, \ldots, 7$

The HDPC applies each voltage vector $\left(\boldsymbol{u}_{s 0}, \boldsymbol{u}_{s 1}, \ldots, \boldsymbol{u}_{s 7}\right)$ in (1) to the model of the PMSM, evaluating its effect and selecting the only one that best fits the system constraints and requirements. $\boldsymbol{u}_{s 0}$ and $\boldsymbol{u}_{s 7}$ are zero-voltage vectors, so that the number of possible states reduces to $N_{s}=7$.

\subsection{The plant model}

The time-domain equations of a PMSM, expressed in the $d q$ rotating reference frame synchronous to the rotor PM, are the following:

$u_{d}=R i_{d}+L_{d} \frac{d i_{d}}{d t}-\omega_{m e} L_{q} i_{q}$
$u_{q}=R i_{q}+L_{q} \frac{d i_{q}}{d t}+\omega_{m e} L_{d} i_{d}+\omega_{m e} \Lambda_{m g}$

where $u_{d}$ and $u_{q}$ are the voltages applied to the $d$ and $q$ axes respectively, $i_{d}$ and $i_{q}$ are the stator currents, $R$ is the phase stator resistance, $L_{d}$ and $L_{q}$ are the synchronous inductances, $\Lambda_{m g}$ is the rotor flux linkage generated by the magnets, $\omega_{m e}=p \omega_{m}$ is the electromechanical speed and $p$ is the number of pole pairs. The electromagnetic and load torque balance equations complete the description of the system:

$$
\begin{aligned}
& \tau=\frac{3}{2} p \Lambda_{m g} i_{q}+\frac{3}{2} p\left(L_{d}-L_{q}\right) i_{d} i_{q} \\
& \tau=J_{m} \frac{d \omega_{m}}{d t}+B_{m} \omega_{m}+\tau_{L}
\end{aligned}
$$


where $J_{m}$ is the total inertia (motor and load) and $B_{m}$ the equivalent viscous friction.

In view of the digital implementation, the time-continuous model of the PMSM is discretised, assuming that both the phase currents and the mechanical position are sampled at multiples of the sampling period $T_{c}$, and defining the integral mean value by the auxiliary operator:

$\langle x\rangle_{k}=\frac{1}{T_{c}} \int_{k T_{c}}^{(k+1) T_{c}} x(t) d t$

The time integration of (2) in the sampling interval $[(k-$ 1) $T_{c}, k T_{c}$ ] returns:

$$
\begin{gathered}
i_{d, k}=i_{d, k-1}+\frac{T_{c}}{L_{d}}\left(L_{q}\left\langle\omega_{m e} i_{q}\right\rangle_{k-1}-R\left\langle i_{d}\right\rangle_{k-1}+\left\langle u_{d}\right\rangle_{k-1}\right) \\
i_{q, k}=i_{q, k-1}+\frac{T_{c}}{L_{q}}\left(-L_{d}\left\langle\omega_{m e} i_{d}\right\rangle_{k-1}-R\left\langle i_{q}\right\rangle_{k-1}\right. \\
\left.+\left\langle u_{q}\right\rangle_{k-1}-\Lambda_{m g}\left\langle\omega_{m e}\right\rangle_{k-1}\right)
\end{gathered}
$$

After some algebraic manipulations, and assuming constant voltage and speed within each sampling period (i.e. $\left\langle\omega_{m e}\right\rangle_{k-1} \approx \omega_{m e, k-1}$ and $\left\langle u_{d}\right\rangle_{k-1}=u_{d, k-1}, \quad\left\langle u_{q}\right\rangle_{k-1}=$ $\left.u_{q, k-1}\right)$, the averaged currents are expressed as:

$\left\langle i_{d}\right\rangle_{k-1} \approx \frac{i_{d, k-1}+i_{d, k}}{2}$
$\left\langle i_{q}\right\rangle_{k-1} \approx \frac{i_{q, k-1}+i_{q, k}}{2}$

while the coupling terms are:

$\left\langle\omega_{m e} i_{d}\right\rangle_{k-1} \approx \omega_{m e, k-1}\left\langle i_{d}\right\rangle_{k-1}$

$\left\langle\omega_{m e} i_{q}\right\rangle_{k-1} \approx \omega_{m e, k-1}\left\langle i_{q}\right\rangle_{k-1}$

The relationship (6) can be rewritten in a state-space form as:

$\boldsymbol{i}_{d q, k}=\boldsymbol{A}_{k-1} \boldsymbol{i}_{d q, k-1}+\boldsymbol{g}_{k-1}$

where $\boldsymbol{i}_{d q, k}=\left[\begin{array}{ll}i_{d, k} & i_{q, k}\end{array}\right]^{T}$ is the current vector in $k T_{c}$ and the matrices $\boldsymbol{A}_{k-1}$ and $\boldsymbol{g}_{k-1}$ contain the other terms of the equation (6). The right-hand side of the equation (9) depends only on measured quantities at the instant $(k-1) T_{c}$, namely the currents $\boldsymbol{i}_{d q, k-1}$, the voltages $\boldsymbol{u}_{d q, k-1}$ and the speed $\omega_{m e, k-1}$. Therefore, it returns the currents prediction $\hat{\boldsymbol{\imath}}_{d q, k \mid k-1}$ in $k T_{c}$ :

$\hat{\boldsymbol{\imath}}_{d q, k \mid k-1}=\boldsymbol{A}_{k-1} \boldsymbol{i}_{d q, k-1}+\boldsymbol{g}_{k-1}$

The prediction model is completed by the electro-mechanical speed prediction obtained from (4):

$\widehat{\omega}_{m, k \mid k-1}=\left(1-\frac{T_{c} B_{m}}{J_{m}}\right) \omega_{m, k-1}+\frac{T_{c}}{J_{m}}\left(\hat{\tau}_{k-1}-\hat{\tau}_{L}\right)$

The estimated torque $\hat{\tau}_{k-1}$ is derived from (3) by substituting the measurements at $k T_{c}$. The speed prediction in (11) is then: $\widehat{\omega}_{m, k \mid k-1}=f_{\omega}\left(\omega_{m, k-1}, \hat{\boldsymbol{\imath}}_{d q, k \mid k-1}, \hat{\tau}_{L}\right)$

It is worth to note that the average current measurements at step depend on the voltages at step $(k-2) T_{c}$, resulting in a two-step delay between voltage application and speed variation.

\subsection{Load torque estimation}

The load torque $\hat{\tau}_{L}$ is estimated by a first-order disturbance observer (DOB) shown in Figure 1.

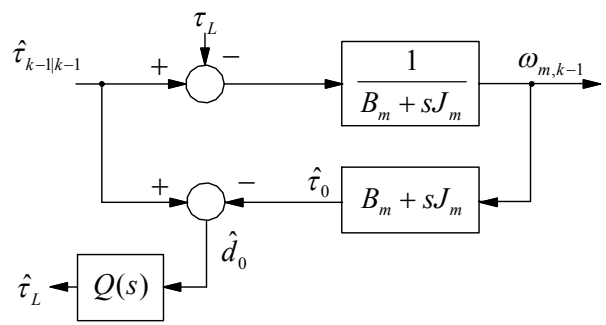

Figure 1: DOB general schematic.

The DOB uses the difference between actual and model speed as an equivalent disturbance applied to the (3), by considering a slowly-variable load torque (i.e. $d \tau_{L} / d t \approx 0$ ). The DOB feedback $\hat{d}_{0}$ cannot be obtained by reversing the mechanical model of the system, since it obviously results in an improper function. An additional transfer function $Q(s)$, with relative degree $\rho_{Q}$ greater or equal to the relative degree of the model plant, is inserted. Choosing a first-order low-pass filter of the type $Q(s)=1 /\left(1+s \tau_{Q}\right)$, the dynamic of the DOB is:

$\hat{\tau}_{0, k-1}=J_{m} \frac{\omega_{m, k-1}-\omega_{m, k-2}}{T_{c}}+B_{m} \omega_{m, k-1}$
$\hat{d}_{0, k-1}=\hat{\tau}_{k-1}-\hat{\tau}_{0}$
$\hat{\tau}_{L, k}=\frac{\tau_{Q}}{\tau_{Q}+T_{C}} \hat{\tau}_{L, k-1}-\frac{T_{c}}{\tau_{Q}+T_{c}} \hat{d}_{0, k-1}$

The cut-off frequency $f_{Q}=1 /\left(2 \pi \tau_{Q}\right)$ is a trade-off between observer dynamic and system noise. In this work, a frequency $f_{Q}=10 \mathrm{~Hz}$ was considered.

\section{The control decisional structure}

The target is the selection of the best voltage vector (control input) to be applied at each sampling instant. The optimal choice is inherently embedded into the design of the HDPC structure. Figure 2 reports the timing diagram of the procedure. For the sake of clarity, the length of a single rectangle is magnified and it is not proportional to the actual execution time. The initialization starts with the measurement of the phase currents, of the speed and the bus voltage $U_{D C}$. Assuming to be at instant $(k-1) T_{c}$, the routine estimates the currents at time instant $k T_{c}$ (by (10)) and the speed at time instant $(k+1) T_{c}$ (by (12)). The estimation is performed by using also the voltages $u_{d, k}$ and $u_{q, k}$ computed in the previous time instant $(k-2) T_{c}$. 


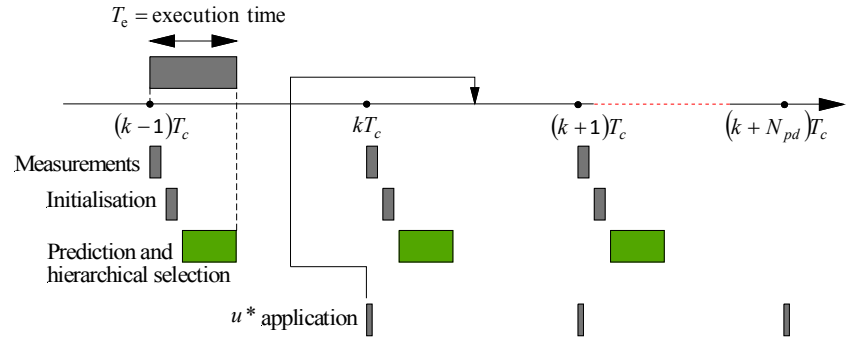

Figure 2: Timing diagram of the HDPC control execution.

The next routine (Figure 2) performs the one-step prediction of the currents and speed by testing in sequence (10) and (12) with all of the $N_{s}$ available voltage configurations. The hierarchical decisional structure is summarised in Figure 3. The highest priority is given to the minimisation of the speed error under mandatory current limits. A voltage vector is accepted only if the predicted current trajectory is within the nominal boundary for the whole prediction horizon $N_{p d}$. For each of the valid voltage vectors, the routine predicts the speed error at the time instant $\left(k+N_{p d}+1\right) T_{c}$, assuming a constant speed reference $\omega_{m}^{*}$. A new set is composed by those voltage vectors that induce a speed error within a predefined boundary $e_{L}$. In case of a void set, the drive is considered under transient and the optimal voltage is the one giving the minimum speed error:

$e_{\omega m}^{s}=\left|\widehat{\omega}_{m}-\omega_{m}^{*}\right|$

$s^{*}=\min _{s \in N_{s}}\left\{e_{\omega m}^{s}\right\}$

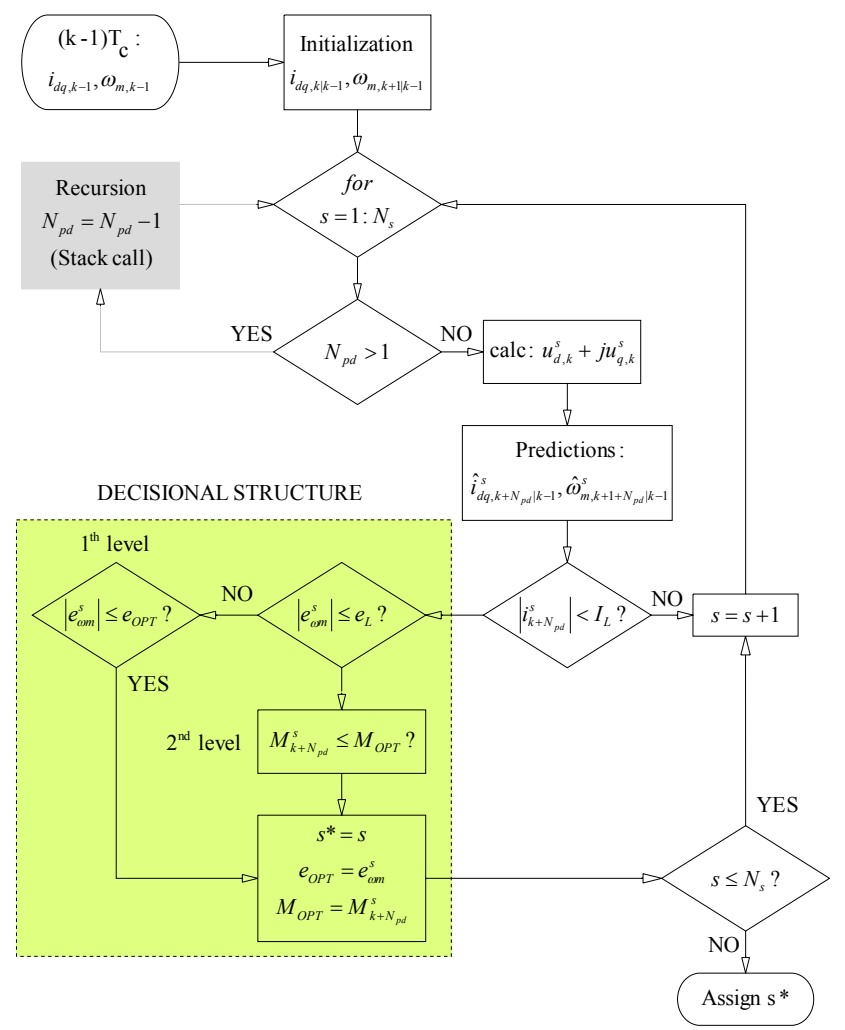

Figure 3: Time evolution of the HDPC control actions.
It is worth to note that in case of a resulting voltage set composed by a single voltage element, no further selection is possible and the hierarchical procedure is concluded. Otherwise, the second hierarchical level is executed with the target of reaching the MTPA condition, derived by considering a polar notation for the currents $i_{d}$ and $i_{q}$ [12]:

$M_{k+N_{p d}}^{s}=\hat{\imath}_{d, k+N_{p d}}^{s}+\frac{\left(L_{d}-L_{q}\right)\left[\left(\hat{\imath}_{d, k+N_{p d}}^{s}\right)^{2}+\left(\hat{\imath}_{q, k+N_{p d}}^{s}\right)^{2}\right]}{\Lambda_{m g}}$
$s^{*}=\min _{s \in N_{s}}\left\{M_{k+N_{p d}}^{s}\right\}$

In general, the optimal trajectory is calculated over a prediction horizon of $N_{p d}$ steps. The hierarchical procedure is recursively executed at each step, so that the number of possible voltage vector sequences grows exponentially with $N_{p d}$. Since this has a negative impact on the implementation, an investigation has been performed in order to understand its optimal value. No performance benefits have been observed unless $N_{p d}$ is large enough to include the mechanical transient of the system. On the other end, such large values of $N_{p d}$ are an excessive computational load even for powerful FPGAs.

With reference to (15), the optimal voltage vector $s^{*}$ satisfying the hierarchical restrictions is applied between $k T_{c}$ and $(k+1) T_{c}$. It is worth to note that the algorithm reaches automatically the MTPA condition regardless of the machine rotor structure (either isotropic or anisotropic), due to the inherent current vector minimisation capability.

\subsection{The HDPC with augmented state vectors set}

Conventional DPC schemes avoid the use of modulation techniques [4], restricting the control input to the eight state voltage vectors. The reduced set causes high ripple at steady state states, which can be mitigated only by a higher switching frequency. It is worth to note that many papers on finite-state DPC do not present results obtained at a full industrial DC-link bus voltage of $560 \mathrm{~V}$, due the unacceptable high current ripple. With the aim of reducing the current ripple without undermining the simplicity of the finite-state solution, this paper explores the use of six additional voltage vectors allocated at $/ 3+\pi / 6 \quad(k=1, \ldots, 6)$. They are generated by applying two adjacent discrete voltage vectors for half of the sampling period [9].

As a distinctive feature of the proposed control algorithm, the amplitude of the voltage vector can be reduced by a coefficient $0<k_{M} \leq 1$, at lower speeds, when the backelectromotive force is lower and less voltage is required for reaching the control targets. This adds a degree of freedom in the control action, improves the ripple content while keeping the prediction stage limited to $N_{s}=14$ voltage vectors.

\section{FPGA implementation}

The proposed HDPC was implemented in a Xilinx ML605 board with a Virtex 6 FPGA. The design process was 
developed with the Xilinx System Generator toolbox in the Matlab/Simulink environment. The input signals were properly scaled, in order to obtain a fixed-point integer representation of the model. For the data logging of the variables, a custom Matlab user interface was created. Some internal variables of the HDPC were stored into the FPGA in First-In First-Out (FIFO) buffers linked to the Matlab interface. Figure 4 shows that each VHDL routine is enabled separately by a finite-state machine (FSM) configured to provide a correct timing of the operations. The compiled Simulink/Matlab project was directly downloaded into the ML605 by using a USB/JTAG interface module.

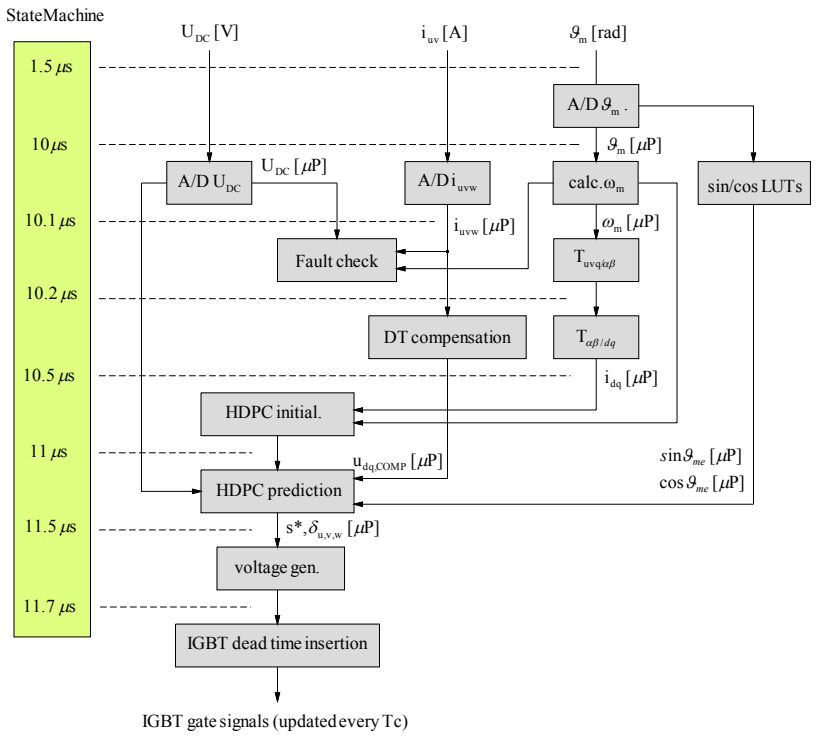

Figure 4: Timing diagram of the FPGA-based HDPC.

The FSM is synchronized with a main counter, that counts from zero to $T_{c} / T_{s}$ ( $T_{s}=5 \mathrm{~ns}$ is the FPGA clock period), and at predefined time instants a short pulse is issued to activate the routines in sequence. To reduce the execution time, the current and DC-link bus voltage measurement, the speed calculation and the $\sin / \cos$ calculation of the electromechanical position are performed in parallel. As shown in Figure 4, the first task is the A/D conversion, where the currents and the DC bus are acquired with 12-bit resolution and the absolute position is recorded with 14-bit resolution. The measured currents are converted in the $d q$ reference frame $\left(T_{u v w / \alpha \beta}\right.$ and $T_{\alpha \beta / d q}$ routines in Figure 4) while the voltage distortion caused by the inverter dead time (DT) effects and the IGBTs nonlinearities are compensated as described in [10].

The FPGA area occupied by the proposed control strategy was investigated. From the summary file of the compilation, it was shown that the project occupied $10 \%$ of the available slices and $61 \%$ of the DSP48E1 slices (used for wide math functions, DSP filters, and complex arithmetic without the use of general FPGA logic). Many connections were produced mainly due to the programming approach, which optimised the fast execution of the prediction algorithm (with a parallelisation of the prediction for the 14 voltage vectors obtained at once) penalising the FPGA resources, the other term of the design trade-off. In case of less area occupation is desired, the project could be reprogrammed exploiting the prediction model recursively. However, that solution would impose a limitation on the maximum number of predictions, which is approximately given by the ratio between the switching period and the time execution of one prediction step.

A test bench composed by two 1.5-kW PMSMs connected in back-to-back configuration with a torque meter mounted on the shaft was used to test the HDPC algorithm. The PMSM used as a load machine was fed by a $4-\mathrm{kW}$ inverter, while the PMSM under test was driven by a customised inverter that received the control signals from the FPGA.

The proposed implementation ensures an execution time of $T_{e}=11.7 \mu \mathrm{s}$, with the bottleneck in the $\mathrm{A} / \mathrm{D}$ conversion, that requires approximately $10 \mu \mathrm{s}$. Faster A/D converters would surely reduce the total execution time of the HDPC, which has anyway a lower bound represented by the maximum allowed switching frequency of the inverter. In this work, a sampling time of $10 \mathrm{kHz}$ was selected.

\section{Simulation and experimental results}

The block schematic of the complete HDPC drive system is shown in Figure 5.

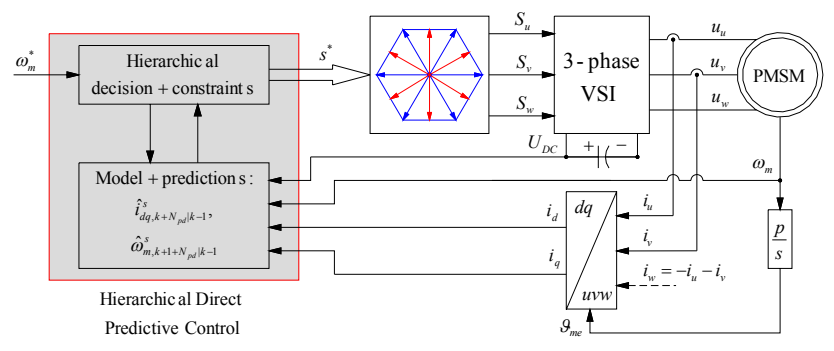

Figure 5: Block diagram of the structure of the HDPC with $N_{s}=14$ voltage vectors

The PMSM in Table 1 has been considered for the simulation tests, with the control parameters of Table 2. Figure 6 shows a speed step variation followed by two consecutive torque increments of half the nominal torque each.

\begin{tabular}{|c|c|}
\hline Motor parameter & Value \\
\hline Ph-ph nominal voltage $U_{N}$ & $400 \mathrm{~V}_{\text {eff }}$ \\
Polar pairs $p$ & 2 \\
Nominal power $P_{N}$ & $1.5 \mathrm{~kW}$ \\
Saliency ratio $\xi=L_{q} / L_{d}$ & $\approx 7$ \\
\hline
\end{tabular}

Table 1: PMSM parameters. 


\begin{tabular}{|c|c|}
\hline Control parameter & Value \\
\hline Switching frequency $f_{s}$ & $10 \mathrm{kHz},\left(T_{c}=100 \mu \mathrm{s}\right)$ \\
State voltages $N_{s}$ & 14 \\
Speed boundary $e_{L}$ & $0.001 \div 0.01 \omega_{N}$ \\
Current limit $I_{L}$ & $0.8 \div 1.5 I_{N}$ \\
DC link voltage $U_{D C}$ & $560 \mathrm{~V}$ \\
Modulation index $k_{M}$ & $0.3 \div 1$ \\
\hline
\end{tabular}

Table 2: HDPC algorithm parameters.
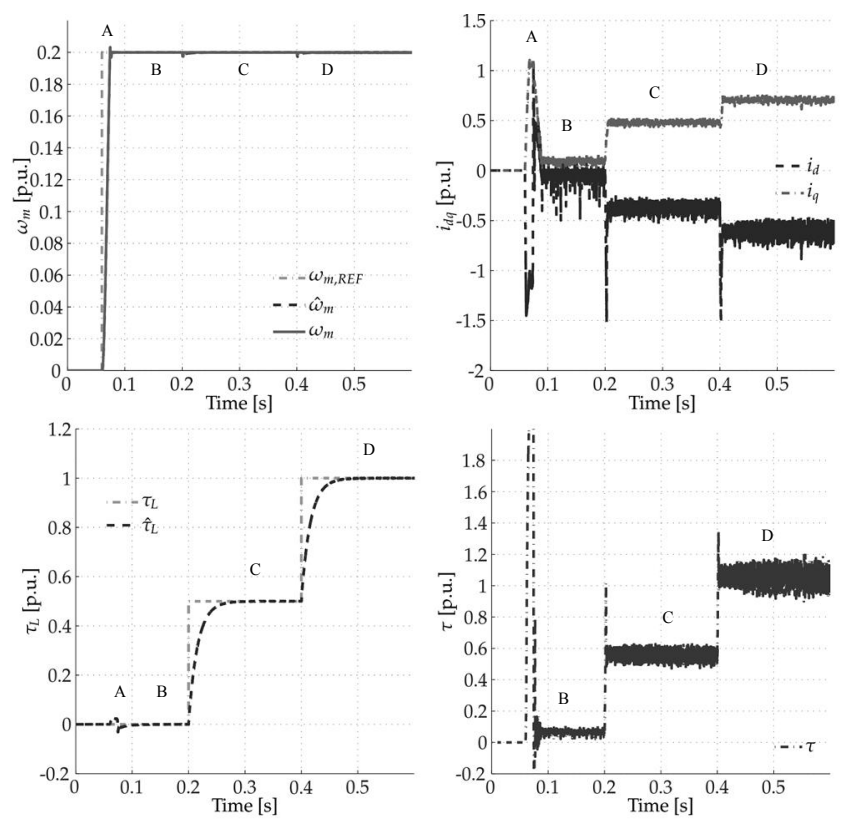

Figure 6: Speed, current, torque and DOB response in the simulation case.

During the speed transient (interval A) the motor is controlled exclusively by the first hierarchical level, since the speed error does not fulfil the $e_{L}$ boundary condition. Once reached the rated speed ( $e_{L}$ boundary fulfilled, interval $\mathrm{B}$ ), the torque reaches a steady-state condition. The steady-state condition C and $\mathrm{D}$ follow the two positives load torque steps of $\tau_{N} / 2$. Again, during these transients the control operates using the first hierarchical level only. At steady-state, the zero speed error testified the correct operation of the load torque observer.

The left graph in Figure 7 shows the current states in the $i_{d}-i_{q}$ plane and the theoretical MTPA curve of the motor (dashed line) [12], showing that the control action converges to the MTPA condition during the steady-state speed regions of Figure 6.

The right graph in Figure 7 shows the average switching frequency $\bar{f}_{s w}$ of the HDPC during the operation of Figure 6 , given by the moving average of switch transitions of the IGBTs computed on a time window of $N_{T_{C}}=250 \cdot T_{c}$. As expected, the HDPC has a variable switching frequency, with the upper bound set by the sampling frequency $f_{s w}$. This is different with respect to the conventional modulation techniques (e.g. the space-vector modulation) in which the frequency is fixed.
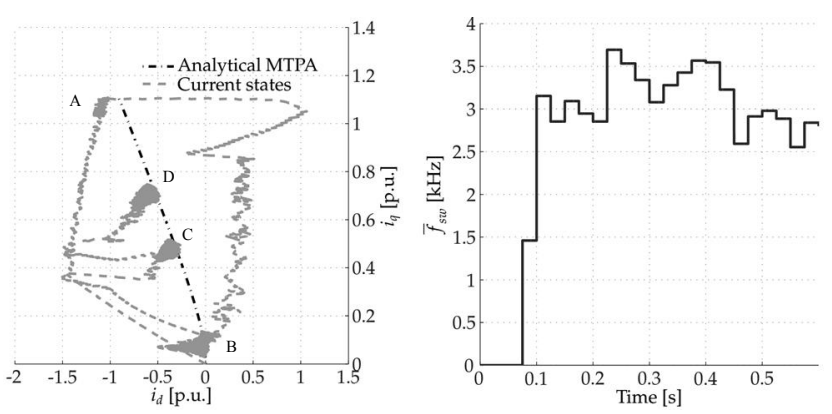

Figure 7: MTPA loci and average switching frequency $\bar{f}_{s w}$.

Due to intrinsic non-linearity of the proposed control algorithm, a direct comparison of its dynamic performances with respect to a conventional PI-cascade control is quite difficult. A possibility is to set a small reference speed variation at no-load condition, in order to perform a smallsignal evaluation similar to the one applicable to the case of field-oriented control. With this method, the simulations have shown a small-signal speed rise time of approximately $2 \mathrm{~ms}$.

Opposite to the simulation case, the experimental measurements were carried out on a PMSM with reduced anisotropy. The speed and current profiles are reported in Figure 8.
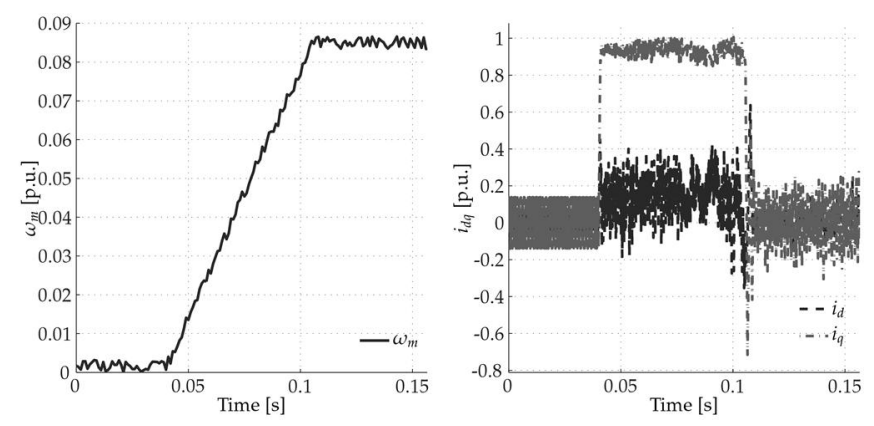

Figure 8: Experimental speed and current transient.

To test the inherent limiting capabilities of the HDPC, the speed step was applied to a high-inertia load (10 times the rotor inertia). The voltage vectors were scaled to $20 \%$ of their nominal value. The result is a speed ramp caused by the constant torque, while the current remains limited to its nominal value (Figure 8 on the right). As expected, the d-axis current component remains quite low, as the MTPA curve lies approximately on the $\mathrm{q}$ axis. It is worth to note that the current ripple is confined to the $10 \%-15 \%$ of the nominal current, with a full DC-link bus voltage $U_{D C}$ of $560 \mathrm{~V}$. Of course, the ripple effect is less evident in the speed measurement, due again to the presence of the high-inertia load. 


\section{Conclusions}

A combined speed-and-current direct predictive control for PMSMs was presented. A particular decisional structure was designed. The highest priority in the hierarchy is given to the speed error minimisation, while a second target aims to the operation in MTPA conditions for reduced copper losses at steady state. With this structure, the current limitation is straightforwardly included in the hierarchical decision process. The proposed scheme is capable of operating in industrial conditions with full DC-link voltages, by resizing the amplitude of the optimal vectors. The ripple is further reduced by increasing from 7 to 14 the candidate voltage vectors. Simulation and experiments performed with a FPGAcontrolled inverter proved the feasibility of the proposed algorithm.

\section{References}

[1] J. Rodriguez, M. Kazmierkowski, J. Espinoza, P. Zanchetta, H. Abu-Rub, H. Young, and C. Rojas, "State of the art of finite control set model predictive control in power electronics," IEEE Trans. Ind. Inf., vol. 9, no. 2, pp. 10031016, 2013.

[2] J. Rodriguez, J. Pontt, C. Silva, P. Correa, P. Lezana, P. Cortes, and U. Ammann, "Predictive current control of a voltage source inverter," IEEE Trans. Ind. Electron., vol. 54, no. 1, pp. 495-503, 2007.

[3] P. Cortes, M. Kazmierkowski, R. Kennel, D. Quevedo, and J. Rodriguez, "Predictive control in power electronics and drives," IEEE Trans. Ind. Electron., vol. 55, pp. 4312-4324, 2008.

[4] M. Kazmierkowski and L. Malesani, "Current control techniques for three-phase voltage source PWM converters: A survey," IEEE Trans. Ind. Electron., vol. 45, no. 5, pp. 691703, Oct. 1998.

[5] J. Holtz and S. Stadtfeld, "A predictive controller for the stator current vector of ac machines fed from a switched voltage source," in Proc. of the IPEC'83, 1983, pp. 16651675.

[6] A. Linder and R. Kennel, "Model predictive control for electrical drives," in Proc. of the PESC'05, 2005, pp. 17931799.

[7] X. Lin-Shi, F. Morel, A. M. Llor, B. Allard, and J. M. Retif, "Implementation of hybrid control for motor drives," IEEE Trans. Ind. Electron., vol. 54, no. 4, pp. 1946-1952, 2007.

[8] E. Schrijver and J. D. van, "Disturbance observers for rigid mechanical systems: equivalence, stability, and design," Journal of Dynamic Systems, Measurement, and Control, vol. 124, no. 4, pp. 539-548, 2002.

[9] S. Vazquez, J. Leon, L. Franquelo, J. Carrasco, O. Martinez, J. Rodriguez, P. Cortes, and S. Kouro, "Model predictive control with constant switching frequency using a discrete space vector modulation with virtual state vectors," in Proc. of ICIT'09, 2009, pp. 1-6.

[10] A. Munoz and T. Lipo, "On-line dead-time compensation technique for open-loop PWMVSI drives,"
IEEE Trans. Power Electron., vol. 14, no. 4, pp. $683-689$, Jul. 1999.

[11] M. Carraro and M. Zigliotto, "Hierarchical direct predictive control of PMSM drives," in Proc. of PRECEDE'11, 2011, pp. 3 -9.

[12] R. Antonello, M. Carraro, and M. Zigliotto, "Towards the automatic tuning of MTPA algorithms for IPM motor drives," in Proc. of ICEM'12, 2012, pp. 1121 -1127. 